\title{
An Abscopal Response to Radiation Therapy in a Patient with Metastatic Non-Small Cell Lung Cancer: A Case Report
}

Kanako Katayama ${ }^{1}$, Akihiro Tamiya ${ }^{1^{*}}$, Taro Koba ${ }^{1}$, Shoichi Fukuda ${ }^{2}$ and Shinji Atagi ${ }^{3}$

${ }^{1}$ Department of Internal Medicine, Kinki-Chuo Chest Medical Center, Osaka, Japan

${ }^{2}$ Department of Radiology, Kinki-Chuo Chest Medical Center, Osaka, Japan

${ }^{3}$ Department of Thoracic Oncology, Kinki-Chuo Chest Medical Center, Osaka, Japan

\begin{abstract}
Introduction: The Abscopal effect refers to radiotherapy-induced tumor regression in lesions distant from a targeted site, and is a rare phenomenon in patients receiving local radiotherapy. This report is the first to describe an Abscopal response in a chemotherapy-naïve non-small cell lung cancer (NSCLC) patient following whole-brain radiotherapy as well as palliative radiotherapy.

Case presentation: A 63-years-old man who was a current-smoker (with 86 pack years) with metastatic NSCLC underwent whole brain radiotherapy (WBRT) plus boost radiotherapy to total dose of 45 Gy in 15 fractions because the metastatic brain tumor with cerebral oedema from the left temporal lobe to the occipital lobe rapidly progressed after the enucleation of the brain tumor. The patient also received palliative radiation (30 Gy in 10 fractions) for the third lumbar vertebral metastasis. The tumor in the left upper lobe of the lung and his mediastinal lymph nodes had regressed in size upon reviewing his follow-up CT results seven weeks post-radiotherapy.
\end{abstract}

Conclusion: The Abscopal effect in metastatic NSCLC patients can occur after the irradiation of metastatic lesions without chemotherapeutic or immunotherapeutic interventions.

Keywords: Non-small cell lung cancer; Abscopal response; Radiotherapy

\section{Introduction}

The Abscopal effect refers to radiation-induced tumor regression in lesions that are distant from a targeted site, and has been recognized for six decades as a rare, unexplained phenomenon in patients receiving local radiotherapy [1]. According to our knowledge, the radiation therapy is not available in patients with multiple metastatic cancer. The abscopal effect is observed outside the treated field of radiation, but it is underrecognized in the clinical practice $[2,3]$. Recent studies have suggested that the Abscopal effect may result from radiotherapyinduced immune system-mediated cancer cell death [4-6]. In support of this hypothesis, the Abscopal effect was reported in a patient who was treated with ipilimumab and fractionated radiotherapy [7]. However, the possibility that ipilimumab alone might be responsible for the patient's response cannot be ruled out, because some non-small cell lung cancer (NSCLC) patients receiving immunotherapeutic agents such as nivolumab achieved good responses and longer progressionfree survival rates $[8,9]$.

We encountered a patient with metastatic NSCLC who experienced the Abscopal effect after whole-brain radiotherapy (WBRT) and palliative radiation for vertebral metastasis in a patient.

\section{Case Presentation}

A 63-year-old man who was a current smoker (40 cigarettes a day for 43 years) presented with worsening dysgraphia and memory impairment. Chest radiography and computed tomography (CT) revealed a $4 \mathrm{~cm}$ solitary tumor in the upper lobe of the left lung with mediastinal lymphadenopathy, and magnetic resonance imaging (MRI) revealed a $3 \mathrm{~cm}$ solitary tumor, assumed to be a metastatic lesion, with cerebral edema extending from the left temporal lobe to the occipital lobe. Bronchoscopic cytology from the lung tumor revealed malignant cells that were consistent with NSCLC. Bone scintigraphy with ${ }^{99 m} \mathrm{Tc}$-methylene diphosphonate demonstrated intense uptake in the third lumbar vertebra. The patient was thus diagnosed with NSCLC, cT2aN2M1b, stage IV. Because his symptoms progressed rapidly, he underwent enucleation of the brain tumor as initial treatment. Pathological examination of the excised cranial lesion confirmed it to be an NSCLC metastasis. Immunohistochemistry revealed positive expression of TTF-1 and CK7, whereas CK20, CA19-9, and CDX2 were negative. Tumor-infiltrating lymphocytes were not observed extensively; however, the brain metastasis progressed immediately after the surgery, and WBRT was therefore performed with a total of $45 \mathrm{~Gy}$ in 15 fractions ( $30 \mathrm{~Gy} / 10$ fractions as WBRT, and $15 \mathrm{~Gy} / 5$ fractions as boost-radiotherapy). The patient also received palliative radiation (30 Gy in 10 fractions) for the third lumbar vertebral metastasis (Figure 1).

The patient's lung tumor immediately shrank after radiotherapy as observed by radiography. The tumor in the left upper lobe of the lung had regressed in size upon reviewing his follow-up CT results seven weeks post-radiotherapy ( $43 \mathrm{~mm}$ to $26 \mathrm{~mm}: 40 \%$ reduction), as had his mediastinal lymph nodes (Figure 2). After we confirmed the Abscopal effect, the patient received four cycles of cisplatin, gemcitabine, and bevacizumab, and was maintaining a good clinical response at his 9-month follow-up session. At this time, disease progression was observed with an increase in the primary tumor size and multiple pulmonary metastases.

*Corresponding author: Akihiro Tamiya, MD, Department of Interna Medicine, Kinki-Chuo Chest Medical Center 1180 Nagasone-cho, Kita-Ku, Sakai City, Osaka 5918555, Japan, Tel: 81722523021; Fax: 81722511372 ; E-mail: atamiya@kch.hosp.go.jp

Received March 15, 2017; Accepted March 25, 2017; Published March 28, 2017

Citation: Katayama K, Tamiya A, Koba T, Fukuda S, Atagi S (2017) An Abscopal Response to Radiation Therapy in a Patient with Metastatic NonSmall Cell Lung Cancer: A Case Report. J Cancer Sci Ther 9:365-367. doi 10.4172/1948-5956.1000443

Copyright: (c) 2017 Katayama K, et al. This is an open-access article distributed under the terms of the Creative Commons Attribution License, which permits unrestricted use, distribution, and reproduction in any medium, provided the original author and source are credited. 

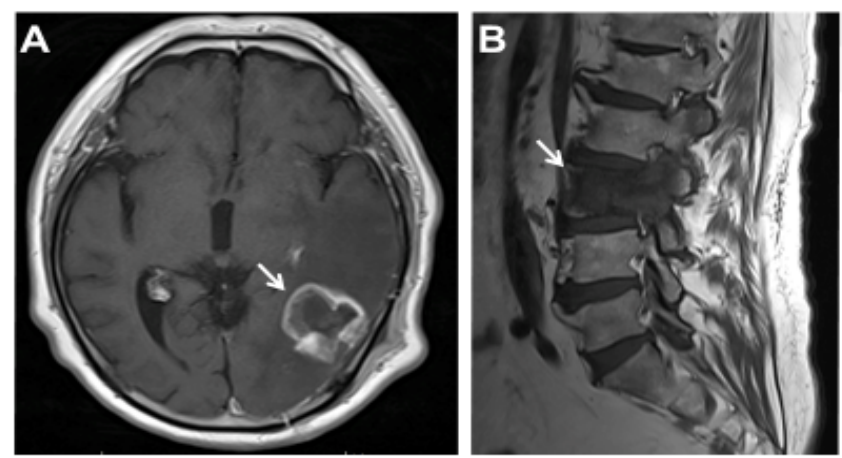

Figure 1: Metastatic sites (arrows) prior to treatment with radiotherapy. A Axial contrast enhanced T1-weighted brain magnetic resonance imaging (MRI) shows a ring-enhancing mass in the left parietal lobe. B: Sagittal contrast enhanced T1-weighted lumbar MRI shows a metastatic spinal tumor at the L3 vertebra.
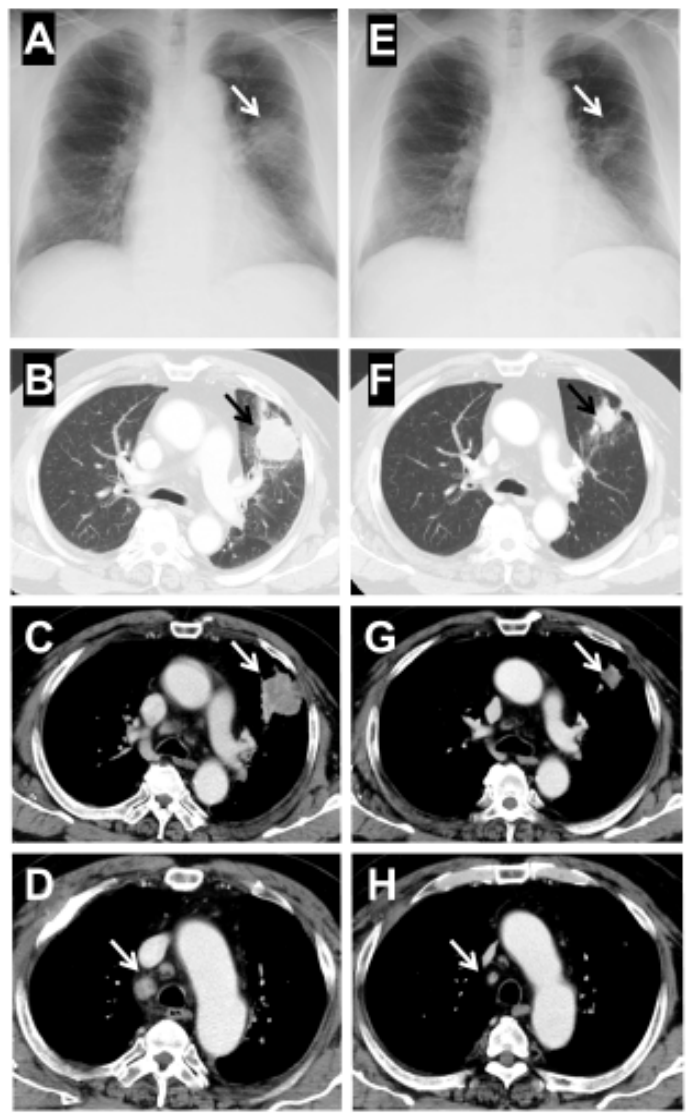

Figure 2: Whole-brain radiotherapy and palliative radiation for vertebral metastasis produced an abscopal response. Chest radiography and transverse computed tomography performed before (left; A-D) and approximately seven weeks after (right; E-H) radiotherapy. The tumor mass in the left upper lung as well as the mediastinal lymph nodes are reduced in size (arrows).

\section{Discussion}

To our knowledge, ours is the first patient with metastatic NSCLC who was reported to exhibit the Abscopal effect after WBRT and palliative radiation without having undergone chemotherapy or immunotherapy.
There are few reports of the Abscopal effect in patients with NSCLC; however, those that involved patients who were treated with radiotherapy alone exhibited reduced metastatic sites after primary site irradiation [10-12]. Our case was distinct in that the Abscopal effect was observed in primary lung tumors directly following the irradiation of metastatic sites, in the absence of intervening immunotherapy.

The cytotoxic effects of radiotherapy have been attributed to double-stranded DNA damage. However, recent studies suggest that the immunomodulatory effects of radiotherapy can contribute to its therapeutic efficacy. Some preclinical experiments have shown that radiotherapy inhibits the mechanisms of tumor immune escape by destroying tumor cells and releasing tumor-associated antigens. These antigens in turn bind antigen presenting cells that then activate tumorspecific cytotoxic $\mathrm{T}$ cells. The antitumor activity of cytotoxic $\mathrm{T}$ cells can be observed in the non-irradiated field. Such Abscopal responses can be achieved by irradiating either primary or metastatic tumors [13-18].

Recently, the Abscopal effect was observed after the initiation of treatment with ipilimumab and fractionated radiotherapy in a patient with chemotherapy-refractory metastatic adenocarcinoma of the lung [7]. As previously mentioned, the Abscopal response has been observed following radiotherapy alone. However, the antitumor function is suppressed by regulatory $\mathrm{T}$ cells and immune checkpoint proteins such as cytotoxic T-lymphocyte-associated protein 4 (CTLA-4), programmed cell death protein 1 (PD-1), and its ligand PD-L1. Immune checkpoint inhibitors can strengthen the antitumor immune function caused by radiotherapy, especially as the latter increases the expression of PD-L1 on tumor cells [19]. In fact, combined blockade of PD-1 and CTLA4 during radiotherapy demonstrated a significant therapeutic effect in both irradiated and non-irradiated large-burden tumors in vivo [20]. However, the possibility that ipilimumab alone might have been responsible for the aforementioned patient's response cannot be ruled out. In contrast, our case showed the occurrence of the Abscopal effect after the irradiation of metastatic lesions without having administered chemo- or immunotherapeutic agents such as ipilimumab. Although our case is rare, it supports the notion that radiotherapy alone can indeed induce the regression of non-irradiated tumors.

A combination of local radiotherapy and immunotherapy might be more effective than immunotherapy alone to improve the outcomes of select patients with metastatic NSCLC, and our patient may serve as a study-worthy example of the Abscopal effect occurring after radiotherapy. Further studies are warranted to clarify the relationship between the Abscopal effect and immunotherapy/radiotherapy in order to better identify patients with NSCLC who will benefit from combination therapy.

\section{Conclusion}

The Abscopal effect in metastatic NSCLC patients can occur after the irradiation of metastatic lesions without chemotherapeutic or immunotherapeutic interventions.

\section{References}

1. Mole RH (1953) Whole body irradiation: Radiobiology or medicine? Br J Radiol 26: $234-241$.

2. Cavanagh W (2009) The Abscopal effect and the prospect of using cancer against itself, prostate cancer research institute, PCRI Insights February. 12.1.

3. Wersäll PJ, Blomgren H, Pisa P, Lax I, Kälkner KM, et al. (2006) Regression of non-irradiated metastases after extracranial stereotactic radiotherapy in metastatic renal cell carcinoma. Acta Oncologica 45: 493-497. 
Citation: Katayama K, Tamiya A, Koba T, Fukuda S, Atagi S (2017) An Abscopal Response to Radiation Therapy in a Patient with Metastatic NonSmall Cell Lung Cancer: A Case Report. J Cancer Sci Ther 9:365-367. doi: 10.4172/1948-5956.1000443

4. Formenti SC, Demaria S (2009) Systemic effects of local radiotherapy. Lancet Oncol 10: 718-726.

5. Formenti SC, Demaria S (2013) Combining radiotherapy and cancer immunotherapy: A paradigm shift. J Natl Cancer Inst 105: 256-265.

6. Ma Y, Kepp O, Ghiringhelli F, Apetoh L, Aymeric L, et al. (2010) Chemotherapy and radiotherapy: Cryptic anticancer vaccines. Semin Immunol 22: 113-124.

7. Golden EB, Demaria S, Schiff PB, Chachoua A, Formenti SC (2013) An Abscopal response to radiation and ipilimumab in a patient with metastatic nonsmall cell lung cancer. Cancer Immunol Res 1: 365-372

8. Brahmer J, Reckamp KL, Baas P, Crino L, Eberhardt WE, et al. (2015) Nivolmab versus docetaxel in advanced squamous-cell non-small cell lung cancer. $\mathrm{N}$ Engl J Med 373: 123-125.

9. Borghaei H, Paz-Ares L, HornL, Spigel DR, Steins M, et al. (2015) Nivolmab versus docetaxel in advanced non squamous non-small cell lung cancer. $\mathrm{N}$ Engl J Med 373: 1627-1639.

10. Siva S, Callahan J, MacManus MP, Martin O, Hicks RJ, et al. (2013) Abscopal effects after conventional and stereotactic lung irradiation of non-small-cell lung cancer. J Thorac Oncol 8: e71-e72.

11. Abuodeh Y, Venkat P, Kim S (2016) Systematic review of case reports on the Abscopal effect. Curr Probl Cancer 40: 25-37.

12. Cong Y, Shen G, Wu S, Hao R (2016) Abscopal regression following SABR for non-small-cell-lung cancer: a case report. Cancer Biol Ther 18: 1-3.
13. Grass GD, Krishna N, Kim S (2016) The immune mechanisms of Abscopal effect in radiation therapy. Curr Probl Cancer 40: 10-24.

14. Takeshima T, Chamoto K, Wakita D, Ohkuri T, Togashi Y, et al. (2010) Local radiation therapy inhibits tumor growth through the generation of tumor-specific CTL: its potentiation by combination with Th1 cell therapy. Cancer Res. 70: 2697-2706.

15. Schaue D, Comin-Anduix B, Ribas A, Zhang L, Goodglick L, et al. (2008) T-cel responses to survivin in cancer patients undergoing radiation therapy. Clin Cancer Res 14: 4883-4890.

16. Lee Y, Auh SL, Wang Y, Burnette B, Wang Y, et al. (2009) Therapeutic effects of ablative radiation on local tumor require $C D 8+T$ cells: Changing strategies for cancer treatment. Blood 114: 589-595.

17. Kalbasi A, June CH, Haas N, Vapiwala N (2013) Radiation and Immunotherapy: A synergistic combination. J Clin Invest 123: 2756-2763.

18. Mansfield AS, Park SS, Dong H (2015) Synergy of cancer immunotherapy and radiotherapy. Aging 7: 144-145.

19. Deng L, Liang H, Burnette B, Beckett M, Darga T, et al. (2014) Irradiation and anti-PD-L1 treatment synergistically promote antitumor immunity in mice. J Clin Invest 124: 687-695.

20. Park SS, Dong H, Liu X, Harrington SM, Krco CJ, et al. (2015) PD-1 restrains radiotherapy-induced abscopal effect. Cancer Immunol Res 3: 610-619. 